Revista de Matemática: Teoría y Aplicaciones 2009 16(2): 231-239

CIMPA - UCR ISSN: 1409-2433

\title{
ASIGNACIÓN DE FRECUENCIAS EN TELEFONÍA CELULAR APLICANDO EL PROBLEMA DE COLORACIÓN ROBUSTA
}

\author{
Pedro Lara-Velázquez* Lizbeth Gallardo-López ${ }^{\dagger}$ \\ Miguel Ángel GutiérRez-Andrade ${ }^{\ddagger}$ \\ Sergio G. DE Los Cobos-Silva ${ }^{\S}$
}

Recibido/Received: 21 Feb 2008 - Aceptado/Accepted: 11 Jul 2008

\begin{abstract}
Resumen
El Problema de Coloración Robusta (PCR), es un problema combinatorio del tipo NP-duro del cual ya se conocen varios algoritmos heurísticos rápidos y eficientes que lo resuelven. En el presente trabajo se plantea el problema de asignación de frecuencias para telefonía celular como un PCR: se proponen algunas instancias con este modelo para una red de celulares y se resuelven estas instancias con un algoritmo GRASP. Los resultados indican que se pueden disminuir las interrupciones intermitentes en el servicio y además aumentar la capacidad de la red en aproximadamente un $25 \%$.
\end{abstract}

Palabras clave: Coloración de gráficas, Coloración Robusta, GRASP, Heurísticas.

\begin{abstract}
The Robust Coloring Problem (RCP) is a NP-Hard Problem for which fast and efficient heuristic algorithms has been developed. In this work we present as a PCR the problem of assignment of frequencies for a cellphone grid. Some instances for this model are proposed and solved using a GRASP algorithm. Evidence shows that the intermittent interruptions in service can be eliminated and the overall capacity can be increased in approximately $25 \%$.
\end{abstract}

*Departamento de Sistemas, División de Ciencias Básicas e Ingeniería, Universidad Autónoma Metropolitana-Azcapotzalco, Av. San Pablo, No. 180, Col. Reynosa Tamaulipas, Del. Azcapotzalco, Ciudad de México, C.P. 02200, México. E-Mail: pedro_lara@correo.azc.uam.mx.

${ }^{\dagger}$ Misma dirección que P. Lara. E-Mail: glizbeth@correo.azc.uam.mx.

${ }^{\ddagger}$ Departamento de Ingeniería Eléctrica, División de Ciencias Básicas e Ingeniería, Universidad Autónoma Metropolitana-Iztapalapa, Av. San Rafael Atlixco, No. 186, Col. Vicentina, Del. Iztapalapa, Ciudad de México, C.P. 09340, México. E-Mail: gamma@xanum.uam.mx.

${ }_{\S}^{\S}$ Misma dirección que M. A. Gutiérrez. E-Mail: cobos@xanum.uam.mx. 
Keywords: Graph coloring, Robust coloring problem, Heuristics, GRASP.

Mathematics Subject Classification: 90B40, 90B80, 90C10.

\section{Introducción}

El PCR es un problema combinatorio, que consiste en la asignación de recursos escasos a un conjunto de usuarios; se distingue del problema de coloración mínima [Diestel, 2000] porque permite obtener soluciones donde no solo es importante encontrar soluciones válidas, sino también que sean estables, es decir, que al hacer modificaciones al problema, la solución obtenida continúe siendo válida con una alta probabilidad [Ramírez, 2000]. Este problema es del tipo NP-duro [Yánez, 2003] y para más de 30 usuarios es necesario utilizar algoritmos de heurísticos. En el caso concreto de la telefonía celular, los usuarios son los teléfonos celulares que están realizando una llamada, y el recurso escaso es la frecuencia a la cual se transmite la señal.

En la segunda sección de este artículo se describe el PCR; la tercera muestra un ejemplo de asignación de frecuencias como una instancia del PCR; la cuarta sección describe el proceso actual de asignación de frecuencias en teléfonos celulares; en la quinta sección se propone un modelo de asignación de frecuencias a teléfonos celulares basado en el PCR; en la sexta sección se muestra una instancia de prueba del modelo anterior, resuelto con un algoritmo glotón y un algoritmo GRASP; finalmente en la última se presentan las conclusiones y las perspectivas de investigación.

\section{El problema de coloración robusta}

El problema de Coloración Mínima (PCM) es un modelo para solucionar problemas donde un conjunto de usuarios compiten por un conjunto limitado de recursos, en un instante de tiempo. Este modelo no supone cambios en el esquema de competencia en instantes posteriores. El PCM considera una gráfica formada por un conjunto de vértices $V$, que representa a los usuarios; un conjunto de colores $C$, que representa a los recursos; y un conjunto de aristas $E$, que representa las incompatibilidades, entre los usuarios. Una incompatibilidad representa la probabilidad de que dos usuarios compitan por un mismo recurso en un momento determinado.

A diferencia del PCM, el PCR es un modelo para problemas donde se compite por recursos en diferentes instantes de tiempo. Este modelo considera dos gráficas, una que llamaremos original o $G$, la cual es idéntica a la utilizada en el PCM, y otra que llamaremos gráfica complementaria o $\bar{G}$, formada por todas las incompatibilidades que podrían ocurrir posteriormente (aristas complementarias) -no incluidas en la gráfica original-, denotadas como $\bar{E}$. Es decir, si las aristas complementarias se definen como:

$$
\{i, j\} \in \bar{E} \Leftrightarrow\{i, j\} \notin E
$$


Entonces el grafo complementario esta formado por:

$$
\bar{G}=(V, \bar{E}) .
$$

A las aristas de la gráfica complementaria les asociamos una penalización, $p_{i j}$ que indica la penalización que hay en la arista complementaria que conecta a los vértices $i$ y $j$. Esta penalización puede indicar, por ejemplo, la probabilidad de que esta arista forme parte de la gráfica original en un futuro cercano. Una coloración en particular que se realiza con $k$ colores se le denota como $C^{k}$. Al color asignado a un vértice $i$ se le denota como $C(i)$. Una propiedad del PCR es la función de rigidez $R\left(C^{k}\right)$, la cual se define como la suma de las penalizaciones de las aristas complementarias cuyos vértices extremos tienen el mismo color:

$$
R\left(C^{k}\right)=\sum_{\{i, j\} \in \bar{E}, C(i)=C(j)} p_{i j} .
$$

Otra propiedad del PCR es que no necesariamente se debe trabajar con la cantidad mínima de colores, ya que el objetivo será optimizar la rigidez y no minimizar el número de colores, en este problema la cantidad máxima de colores que se utilizan, se fija de antemano.

\section{Asignación de frecuencias utilizando PCR}

El PCR se puede utilizar para obtener una asignación de frecuencias de la siguiente forma. Todos los usuarios del espectro electromagnético (estaciones de televisión, radio, telefonía celular, radiocomunicadores, etc.) tienen asignadas ciertas "frecuencias" en las cuales se les permite emitir sus respectivas señales. Por ejemplo, en casi todo el mundo las estaciones de FM pueden transmitir entre 88 y $108 \mathrm{Mhz}$, pero no existen estaciones de FM a una frecuencia menor, porque interferirían con el canal 6 de televisión, y tampoco a una frecuencia mayor, porque interfieren con el canal 7.

Dentro del mismo espectro, cada transmisor debe respetar su ancho de frecuencia, por ejemplo, una estación X de radio FM que transmite a $88.1 \mathrm{MHz}$ tiene derecho de utilizar $0.8 \mathrm{MHz}$ para transmitir sus señales, es decir, no debe existir ninguna transmisión ajena a la transmisión de la estación X en el intervalo de 88.1 a $88.9 \mathrm{MHz}$, la siguiente estación, digamos Estación Y, podría transmitir en el intervalo de 88.9 a 89.7 y así sucesivamente. Siguiendo este razonamiento, en el espacio designado para radio FM, si se utilizan todos los "espacios" disponibles podemos tener a lo más 25 estaciones de FM. Debido a que la señal de transmisión se va atenuando a medida que aumenta la distancia a la estación transmisora, un receptor de FM solamente escucha las estaciones locales y en una ciudad vecina, se puede reutilizar la misma frecuencia para que otra estación en otra ciudad transmita a la misma frecuencia.

Supongamos que 5 equipos de radiocomunicación que están situados en una misma área geográfica, requieren usar alguna frecuencia del espectro electromagnético, con un cierto alcance máximo en sus transmisiones. Los equipos que se encuentran dentro de este alcance máximo podrían competir por la misma frecuencia, por lo que es necesario asignarles diferentes frecuencias. Un ejemplo se muestra en la Figura 1. 

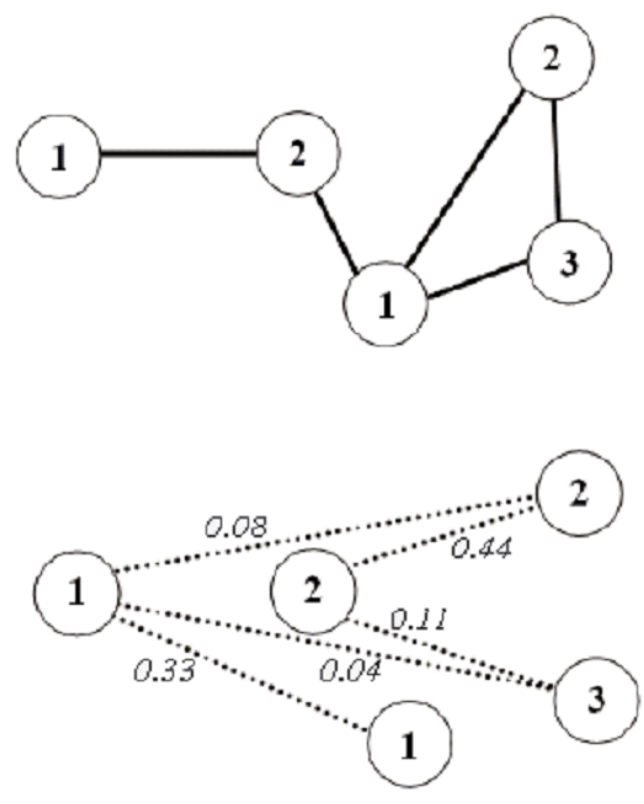

Figura 1: Gráfica original (arriba) y complementaria (abajo) asociadas al problema pintada con tres colores y con rigidez de 0.77 .

En la Figura 1, los vértices representan los equipos de radiocomunicación, los colores (los números dentro de los vértices) representan las frecuencias permitidas para realizar las comunicaciones. Las aristas de la gráfica original indican las incompatibilidades que ocurren al tiempo $t_{1}$. Las aristas de la gráfica complementaria muestran las posibles incompatibilidades que pueden ocurrir en un momento posterior $t_{2}$, con las probabilidades asociadas a cada evento indicadas sobre la arista. En la coloración asociada a estas gráficas, los vértices pintados con el valor "1" aumentan en 0.33 la rigidez asociada al modelo, así como los vértices pintados con el color "2" aumentan en 0.44 la rigidez. Como en este ejemplo no hay otra arista en la gráfica complementaria cuyos vértices tengan el mismo color, la rigidez total sería: $R\left(C^{3}\right)=0.33+0.44=0.77$, este es el valor de rigidez asociado a esta instancia con esta coloración. Otra coloración que también es válida y cuya rigidez es menor $\left(R\left(C^{\prime 3}\right)=0.08+0.11=0.19\right)$, se presenta a en la Figura 2. Si utilizamos cuatro colores podemos encontrar una coloración con rigidez aún menor $\left(R\left(C^{4}\right)=0.04\right)$, vea Figura 3. 

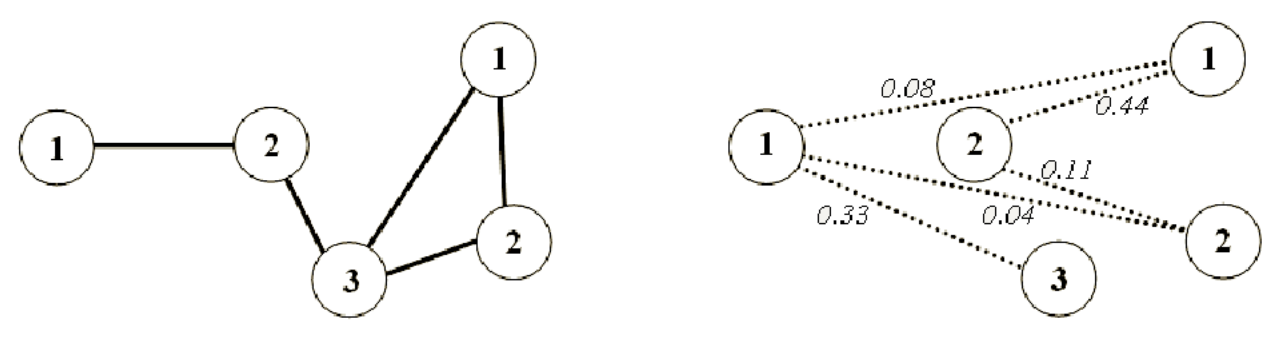

Figura 2: Otra solución con los mismos tres colores con una rigidez de 0.19.
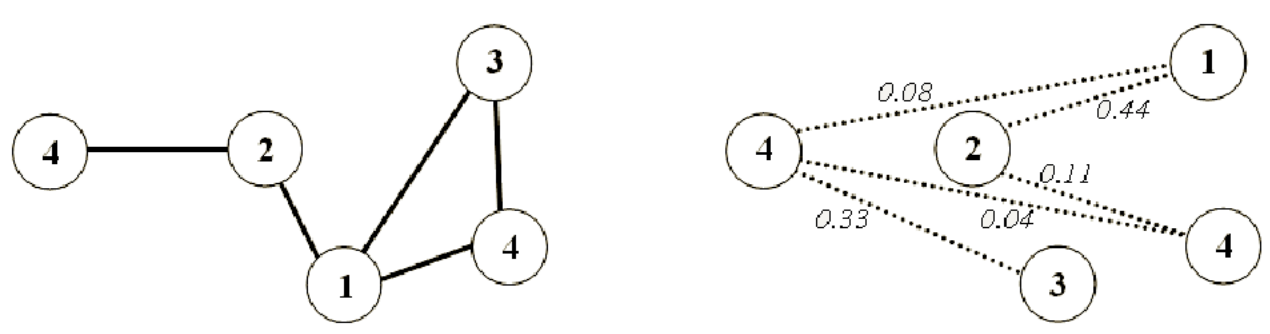

Figura 3: Una solución con cuatro colores y con una rigidez de 0.04.

\section{Forma actual de asignación de frecuencias en teléfonos celulares}

Cuando un persona realiza una llamada a través de un teléfono celular, utiliza dos frecuencias de transmisión, una para hablar (emisión) y otra para escuchar (recepción), la señal de emisión la genera el teléfono celular, y la de recepción la genera una estación que se encuentra a lo más a un par de kilómetros del celular. Esta estación base administra las llamadas que provienen de los celulares en una región geográfica. Esta estación base administra las llamdas que provienen de los celulares que se encuentran dentro de su área geográfica de servicio (también llamda área de cobertura). Esta administración consiste, entre otras actividades, en la asignación de frecuencias, en el manejo de una base de datos que mantiene el registro de todos los usuarios potenciales en la región y en la transmisión de la señal a otras estaciones base.

La región geográfica que cubre cada base es de aproximadamente $26 \mathrm{~km}^{2}$ y suele llamarse "célula". La distancia que separa a dos células es menor a unos 5 kilómetros. Esta corta distancia entre las células tiene dos ventajas: 1) permite que la señal emitida por un teléfono celular sea de baja potencia, lo cual implica un consumo de energía menor y por ende una batería de tamaño pequeño; 2) permite que más usuarios utilicen el servi- 
cio gracias a que las mismas frecuencias son reutilizadas por otras bases suficientemente alejadas.

La telefonía celular cuenta con $1176^{1}$ canales digitales duales para transmitir las llamadas $^{2}$. A fin de impedir que dos estaciones utilicen la misma frecuencia al mismo tiempo, se asigna a cada célula, uno de siete diferentes conjuntos de frecuencias, en un arreglo similar a un panal, donde dos estaciones adyacentes jamás comparten el mismo conjunto. Cada estación base tiene asignados 1176/7=168 canales lo cual implica que 168 personas pueden utilizar su teléfono celular simultáneamente dentro de una misma célula.

Este mismo problema se presenta cuando existen más celulares compitiendo por una frecuencia para establecer una llamada. Por ejemplo, cuando 5 personas están compartiendo por 4 frecuencias; para cada una de ellas, se presentarán lapsos de silencio en la conversación en una quinta parte del tiempo, y la conversación será adecuada en cuatro quintas partes del tiempo. Para solucionar este problema, se recomienda el uso racional de de las frecuencias; así como su reutilización en casos donde los usuarios se encuentren en puntos opuestos de la célula; o bien, utilizar la información histórica de sus llamadas y de sus movimientos para la asignación de las mismas.

\section{Aplicación del PCR en telefonía celular}

Por lo general, en las redes de telefonía se cuenta con 10 usuarios potenciales por cada frecuencia disponible, en un momento determimado (esto se debe a que un usuario no utiliza el teléfono todo el tiempo). Así que para nuestro modelo, podemos considerar 168 frecuencias (colores) y aproximadamente algo menos de 1700 usuarios potenciales, de los cuales, solamente 168 podrán hablar simultáneamente dentro de la célula ${ }^{3}$. Actualmente, debido a la gran cantidad de celulares que existen y a que los costos por llamada han bajado, esta capacidad se ha sobrepasado. Esta situación provoca que las compañías se vean obligadas, en ocasiones, a "compartir frecuencias" (es decir que dos usuarios compartan al mismo tiempo una misma frecuencia), lo cual implica, a su vez, que el servicio se proporcione de manera intermitente o que la llamada se corte en cualquier momento.

Analicemos la siguiente situación: En la Figura 4, se encuentran cuatro usuarios dentro de una misma célula (el hexágono sombreado), el usuario 1 está realizando una llamada y para ello utiliza una frecuencia, observe que en la región dentro del círculo sombredo, la señal de 1 es lo suficientemente intensa como para impedir que el usuario 2 utilice la misma frecuencia. En el anillo que rodea al círculo sombreado, la señal del usuario 1, aunque es débil generaría interferencia si el usuario 3 empleara su misma frecuencia. Sin embargo,

\footnotetext{
${ }^{1}$ De hecho, en México los canales asignados a telefonía son 1248, pero 72 de ellos se utilizan para mensajería y para comunicaciones entre bases de celulares.

${ }^{2}$ En la actualidad, los teléfonos celulares más recientes pueden utilizar otros conjuntos de frecuencias, lo que aumenta la cantidad real de frecuencias (recursos) por asignar. Este hecho no repercute en los cálculos del ejemplo que se está desarrollando.

${ }^{3}$ El fenómeno de saturación de llamadas ocurre frecuentemente después de un temblor considerable: todo el mundo intenta llamar a sus casas o familiares, y tanto los teléfonos fijos como los celulares se encuentran "ocupados".
} 
en la región dentro del hexágono sombreado, fuera de los dos círculos, la señal del usuario 1 es tan débil, de tal manera que un cuarto usuario podría emplear su misma frecuencia sin que ambas señales causen interferencia. Aún cuando los usuarios 1 y 4 están ubicados en la misma célula, ambos se encuentran lo suficientemente alejados como para utilizar la misma frecuencia. Se considera que esta manera de reutilizar las frecuencias optimiza su asignación, permitiendo a más usuarios usar el servicio sin interferencias.

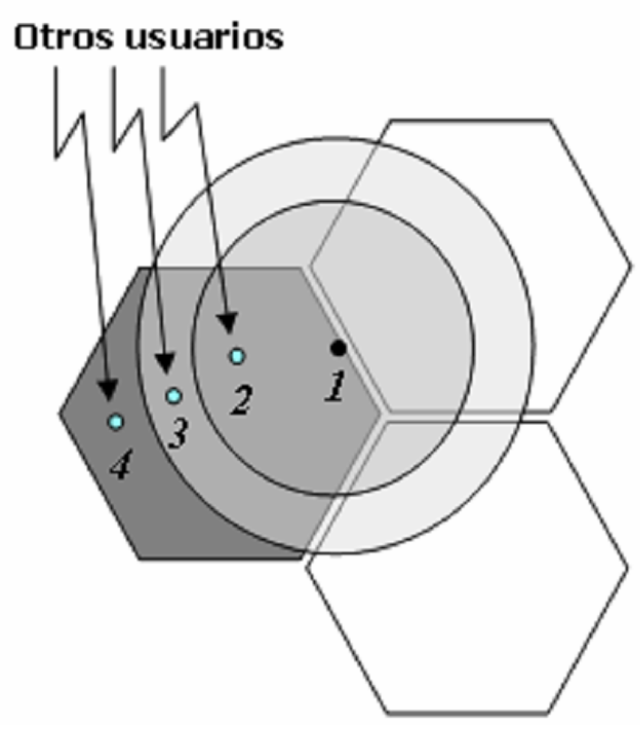

Figura 4: Los tres tipos de interacciones entre usuarios.

La situación anterior se puede modelar como un PCR donde cada usuario se le asigna un vértice de la gráfica. Si dos usuarios se encuentran a una distancia menor a un cierto umbral entonces se asigna una arista en $G$, en caso contrario, cuando dos usuarios se encuentran a una distancia mayor que el umbral se asignará una arista a la gráfica complementaria $\bar{G}$ cuya rigidez está asociada al inverso del cuadrado de la distancia entre ellos (Dado que la intensidad de una señal disminuye con el cuadrado de la distancia que los separa.). Es decir mientras más alejados la penalización por usar la misma frecuencia será menor.

Dadas las coordenadas de los usuarios 1 y 2 , la distancia que los separa es:

$$
d_{1-2}=\sqrt{\left(x_{1}-x_{2}\right)^{2}+\left(y_{1}-y_{2}\right)^{2}}
$$

donde el vector $\left(x_{i}, y_{i}\right)$ indica la localización de cualquier punto $i$ en el espacio.

\section{Instancias de prueba para el modelo}

Para efectos de una simulación, podemos considerar a nuestra célula como una región con radio 1, para la cual la intensidad máxima es un valor unitario. Por lo tanto en este 
modelo, el alcance máximo de la señal se da cuando la distancia es menor o igual a 1 (círculo sombreado de la Figura 4), a partir de esta distancia la señal se va debilitado hasta alcanzar la mitad de su intensidad en $\sqrt{2}$ a partir de ahí se puede considerar como ruido. Es decir, dados dos usuarios, si su distancia está entre cero y $\sqrt{2}$, se prohibe usar la misma frecuencia y si es mayor se asignará como rigidez el inverso del cuadrado de la distancia, es decir, se está tomando el umbral igual a $\sqrt{2}$.

Se generaron en computadora instancias con las propiedades antes mencionadas, para usuarios colocados al azar en una célula. Se tomaron instancias diferentes en donde se colocaron en cada célula 170, 175, 180, y así sucesivamente hasta 210 usuarios. Para cada instancia se obtuvieron dos soluciones, una considerando un algoritmo glotón no robustecido (este algoritmo solo encuentra una solución factible) y un algoritmo GRASP. Para cada número de usuarios distintos se corrieron 10 instancias; que equivalen a una sobre demanda desde un $1 \%$ hasta un $25 \%$ adicional a capacidad máxima.

En la Tabla 1 se presentan los resultados para las 90 instancias consideradas.

\begin{tabular}{cc|cccc}
\hline \hline \# Usuarios & $\begin{array}{c}\text { Usuarios } \\
\text { Extra }\end{array}$ & $\begin{array}{c}\text { Rig. } \\
\text { GRASP }\end{array}$ & $\begin{array}{c}\text { T. Ejec } \\
\text { (seg.) }\end{array}$ & $\begin{array}{c}\text { Rig. } \\
\text { Glotón }\end{array}$ & $\begin{array}{c}\text { T. Ejec } \\
\text { (seg.) }\end{array}$ \\
\hline \multirow{2}{*}{170} & 2 & $1.044 \pm$ & $23.8 \pm$ & $51.4 \pm$ & $8.38 \pm$ \\
& & 0.019 & 1.1 & 2.0 & 0.13 \\
175 & 7 & $3.722 \pm$ & $29.7 \pm$ & $55.4 \pm$ & $8.96 \pm$ \\
& & 0.069 & 1.2 & 3.4 & 0.11 \\
180 & \multirow{2}{*}{12} & $6.462 \pm$ & $31.72 \pm$ & $59.1 \pm$ & $9.66 \pm$ \\
& & 0.088 & 0.56 & 3.3 & 0.26 \\
185 & \multirow{2}{*}{17} & $9.689 \pm$ & $32.98 \pm$ & $58.6 \pm$ & $9.99 \pm$ \\
& & 0.156 & 0.73 & 2.8 & 0.14 \\
190 & 22 & $12.92 \pm$ & $35.71 \pm$ & $57.6 \pm$ & $10.31 \pm$ \\
& & 0.27 & 0.79 & 5.3 & 0.26 \\
195 & 27 & $16.04 \pm$ & $38.19 \pm$ & $61.9 \pm$ & $10.94 \pm$ \\
& & 0.38 & 0.79 & 2.7 & 0.15 \\
200 & \multirow{2}{*}{32} & $19.55 \pm$ & $40.3 \pm$ & $65.3 \pm$ & $11.81 \pm$ \\
& & 0.50 & 1.20 & 5.6 & 0.42 \\
205 & \multirow{2}{*}{37} & $22.86 \pm$ & $44.1 \pm$ & $68.2 \pm$ & $12.24 \pm$ \\
& & 0.93 & 1.1 & 4.5 & 0.17 \\
210 & \multirow{2}{*}{42} & $26.413 \pm$ & $46.53 \pm$ & $73.92 \pm$ & $13.17 \pm$ \\
& & 0.075 & 0.55 & 0.88 & 0.042 \\
\hline \hline
\end{tabular}

Tabla 1: Resultados obtenidos en las 90 instancias generadas.

Con este modelo se encuentran soluciones factibles con una sobre demanda de hasta el $25 \%$ ya sea utilizando el algoritmo GRASP o el glotón. Si se usa el algoritmo GRASP entonces las soluciones son menos susceptibles a cambios en la intensidad de la señal o a movimientos del usuario debido a que tienen una menor rigidez que las asociadas a las soluciones encontradas por el algoritmo glotón (observe en la Tabla 1 las rigideces 
de ambos algoritmos). Por otro lado, los tiempos de ejecución del algoritmo glotón son mucho menores a los del GRASP (alrededor de una tercera parte del tiempo empleado por el GRASP) lo que permite hacer ajustes en tiempo real a la asignación actual.

Cabe recalcar que en cualquiera de los dos casos, siempre se pudo encontrar una coloración válida en la distribución actual, lo que implica que no se tendrían interferencias entre frecuencias en ningún caso, por lo que incluso el esquema del algoritmo glotón (el cual podría convenir por cuestiones de tiempo de ejecución) es más conveniente que el esquema actual de asignación arbitraria.

\section{Conclusiones}

En este trabajo se presenta una aplicación del PCR a la telefonía celular, se presenta también la forma en que se administran los servicios dentro de una célula. Se propone un modelo de coloración robusta para la asignación de frecuencias donde se considera la distancia a la que se encuentran los usuarios entre sí. Se probó el modelo generando un conjunto de instancias en donde se considera una distribución azarosa de los usuarios dentro de la célula. Se comparan dos algoritmos, un algoritmo glotón para el problema de coloración, (es decir, sin robustecer) y otro que considera coloración robusta (algoritmo GRASP), encontrándose que este último es mucho más eficiente con un costo de ejecución menor a un minuto incluso en el caso más grande de usuarios (25\% de sobrecupo). En ambos casos se pudo encontrar una coloración factible (es decir, sin interferencias entre los usuarios), por lo que este esquema es mucho más conveniente que el actual de asignación azarosa, en donde es muy frecuente la interferencia de celulares cuando se tiene sobrecupo.

\section{Referencias}

[1] Diestel, R. (2000) Graph Theory. Springer-Verlag, New York (Electronic Edition).

[2] Ramírez, J. (2000) Extensiones del Problema de Coloración de Grafos. Tesis de Doctorado, Facultad de Ciencias Matemáticas, Universidad Complutense de Madrid.

[3] Yáñez, J.; Ramírez, J. (2003) "The robust coloring problem", European Journal of Operational Research 148(3): 546-558. 